\title{
ON SUBCLASSES OF P-VALENT CLOSE-TO-CONVEX FUNCTIONS
}

\author{
M.K. AOUF
}

Abstract. Let $K[C, D, p, \alpha],-1 \leq D<C \leq 1$ and $0 \leq \alpha<p$ denote the class of functions $g(z)=z^{p}+\sum_{n=p+1}^{\infty} b_{n} z^{n}$ analytic in the unit disc $U=\{z:|z|<1\}$ and satisfying the condition $1+\frac{z g^{\prime \prime}(z)}{g^{\prime}(z)}$ is subordinate to $\frac{p+[p D+(C-D)(p-\alpha)] z}{1+D z}$, $z \in U$. We investigate the subclass of $p$-valent close-to-convex functions $f(z)=$ $z^{p}+\sum_{n=p+1}^{\infty} a_{n} z^{n}$, for which there exists $g(z) \in K[C, D, p, \alpha]$ such that $\frac{p f^{\prime}(z)}{g^{\prime}(z)}$ is subordinate to $\frac{p+[p B+(A-B)(p-\beta)] z}{1+B z},-1 \leq B<A \leq 1$ and $0 \leq \beta<p$. Distortion and rotation theorems and coefficient bounds are obtained.

\section{Introduction}

Let $A_{p}$ ( $p$ a fixed integer greater than zero) denote the class of functions $f(z)=$ $z^{p}+\sum_{k=p+1}^{\infty} a_{k} z^{k}$ which are analytic in $U=\{z:|z|<1\}$. Let $\Omega$ denote the class of bounded analytic functions $w(z)$ in $U$ satisfying the conditions $w(0)=0$ and $|w(z)| \leq|z|$ for $z \in U$.

For $-1 \leq B<A \leq 1$ and $0 \leq \beta<p$, denote by $P[A, B, p, \beta]$ the class of functions $p(z)=p+\sum_{k=1}^{\infty} c_{k} z^{k}$ which are analytic in $U$ and which satisfy that $p(z) \in P[A, B, p, \beta]$ if and only if

$$
p(z) \prec \frac{p+[p B+(A-B)(p-\beta)] z}{1+B z}, \quad z \in U .
$$

By definition of subordination if follows that $p(z) \in P[A, B, p, \beta]$ has a representation of the form

$$
p(z)=\frac{p+[p B+(A-B)(p-\beta)] w(z)}{1+B w(z) .}, \quad w \in \Omega .
$$

The class $P[A, B, p, \beta]$ was introduced by Aouf [1].

Key words and phrases. $p$-Valent, analytic, close-to-convex functions.

1980 Mathematics subject classification codes. $30 \mathrm{C} 45$. 
Given $C, D,-1 \leq D<C \leq 1$ and $0 \leq \alpha<p, K[C, D, p, \alpha]$ and $P^{*}[C, D, p, \alpha]$ denote the classes of functions $f(z) \in A_{p}$ such that $1+\frac{z f^{\prime \prime}(z)}{f^{\prime}(z)} \in P[C, D, p, \alpha]$ and $\frac{z f^{\prime}(z)}{f(z)} \in P[C, D, p, \alpha]$, respectively. The class $P^{*}[C, D, p, \alpha]$ was introduced by Aouf [1]. It follows from the definitions of the classes $K[C, D, p, \alpha]$ and $P^{*}[C, D, p, \alpha]$ that

$$
g(z) \in K[C, D, p, \alpha] \quad \text { if and only if } \quad \frac{z g^{\prime}(z)}{p} \in P^{*}[C, D, p, \alpha]
$$

We note that:

1. $P^{*}[1,-1,1, \alpha]=S^{*}(\alpha), K[1,-1,1, \alpha]=C(\alpha), 0 \leq \alpha<1$, are the well-known classes of starlike functions of order $\alpha$ and convex functions of order $\alpha$, respectively, introduced by Robertson [15].

2. $P^{*}[1,-1, p, \alpha]=S_{p}^{*}(\alpha)$ and $K[1,-1, p, \alpha]=C_{p}(\alpha), 0 \leq \alpha<p$, are, respectively, the class of $p$-valent starlike functions of order $\alpha$, investigated by Goluzina [6] and the class of $p$-valent convex functions of order $\alpha$.

3. $P^{*}[C, D, 1,0]=P^{*}[C, D]$, is the class of functions $f(z) \in A_{1}$, introduced by Janowski [7] and studied further by Goel and Mehrok $[3,4]$ and $K[C, D, 1,0]=K[C, D]$, is the class of functions $f(z) \in A_{1}$, studied by Mazur [13] and Silvia [19].

A function $f(z) \in A_{p}$ is said to be in the class $C[A, B ; C, D, p, \beta, \alpha],-1 \leq A<B \leq$ $1,-1 \leq D<C \leq 1,0 \leq \beta<p$ and $0 \leq \alpha<p$, if there exists $g(z) \in K[C, D, p, \alpha]$ such that

$$
\frac{p f^{\prime}(z)}{g^{\prime}(z)} \in P[A, B, p, \beta]
$$

We note that:

1. $C[1,-1 ; 1,-1,1,0,0]=C$, is the well-known class of close-to-convex functions, introduced by Kaplan [8].

2. $C[A, B, ; C, D, 1,0,0]=C[A, B ; C, D]$, is the class of functions $f(z) \in A_{1}$, studied by Silvia [19].

3. $C[1,-1 ; C, D, 1,0,0]$, was studied by Goel and Mehrok $[4,5]$.

4. $C[1,-1 ; 1,-1,1, \beta, \alpha]=C(\alpha, \beta)$, is the class of close-to-convex functions of order $\alpha$ and type $\beta$, was introduced by Libera [11].

5. In [2] Aouf studied the class $C[1,-1 ; C, D, p, \beta, \alpha]=C[C, D, p, \beta, \alpha]$ of functions $f(z) \in A_{p}$ satisfying

$$
\begin{aligned}
& \frac{z f^{\prime}(z)}{g(z)} \in P[1,-1, p, \beta]=P(p, \beta), \\
& g(z) \in P^{*}[C, D, p, \alpha] .
\end{aligned}
$$




\section{Distortion And Rotation Theorems.}

Unless otherwise mentioned in the sequel, the only restrictions on the real constants $A, B, C, D, \alpha, \beta$ and $p$ are that $-1 \leq D<C \leq 1,-1 \leq B<A \leq 1,0 \leq \alpha<p$ and $0 \leq \beta<p$.

Theorem 1. For $f(z) \in C[A, B ; C, D, p, \beta, \alpha],|z| \leq r<1$,

$$
\begin{aligned}
& r^{p-1} \frac{p-[p B+(A-B)(p-\beta)] r}{1-B r}(1-D r)\left(\frac{C-D}{D}\right)(p-\alpha) \leq\left|f^{\prime}(z)\right| \\
\leq & r^{p-1} \frac{p+[p B+(A-B)(p-\beta)] r}{1+B r}(1+D r) \frac{\left(\frac{C-D}{D}\right)(p-\alpha)}{\left(\frac{P-\alpha}{D}\right)}, D \neq 0, \\
& r^{p-1} \frac{p-[p B+(A-B)(p-\beta)] r}{1-B r} e^{-C(p-\alpha) r} \leq\left|f^{\prime}(z)\right| \\
\leq & r^{p-1} \frac{p+[p B+(A-B)(p-\beta)] r}{1+B r} e^{C(p-\alpha) r}, D=0 .
\end{aligned}
$$

The bounds are sharp.

Proof. For $f(z) \in C[A, B ; C, D, p, \beta, \alpha]$, there exists a $g(z) \in K[C, D, p, \alpha]$ and $p(z) \in P[A, B, p, \beta]$ such that

$$
f^{\prime}(z)=\frac{g^{\prime}(z)}{p} p(z)
$$

Since $g(z) \in K[C, D, p, \alpha]$ if and only if $\frac{z g^{\prime}(z)}{p} \in P^{*}[A, B, p, \alpha]$, for $|z| \leq r<1[1$, Theorem 1]

$$
\begin{aligned}
& p r^{p-1}(1-D r)^{\left(\frac{C-D}{D}\right)(p-\alpha)} \leq\left|g^{\prime}(z)\right| \leq p r^{p-1}(1+D r)^{\left(\frac{C-D}{D}\right)(p-\alpha)}, D \neq 0 \\
& \text { and } \\
& \qquad r^{p-1} e^{-C(p-\alpha) r} \leq\left|g^{\prime}(z)\right| \leq p r^{p-1} e^{C(p-\alpha) r}, D=0
\end{aligned}
$$

Also for $p(z) \in P[A, B, p, \beta]$, we have for $|z| \leq r<1$ [1, Corollary 1]

$$
\frac{p-[p B+(A-B)(p-\beta)] r}{1-B r} \leq|p(z)| \leq \frac{p+[p B+(A-B)(p-\beta)] r}{1+B r} \text {. }
$$

The result follows immediately upon applying (2.3) and (2.2) to (2.1).

Equality is obtained for $f(z) \in C[A, B ; C, D, p, \beta, \alpha]$ satisfying

$$
f^{\prime}(z)=\left\{\begin{array}{l}
z^{p-1}(1+D z)\left(\frac{C-D}{D}\right)(p-\alpha) \cdot \frac{p+[p B+(A-B)(p-\beta)] z}{1+B z}, D \neq 0, \\
z^{p-1} e^{C(p-\alpha) z} \cdot \frac{p+[p B+(A-B)(p-\beta)] z}{1+B z}, D=0
\end{array}\right.
$$


and $z= \pm r$.

Remarks。

1. For $p=1$ and $\alpha=\beta=0$, Theorem 1 agrees with Theorem 1 of Silvia [19].

2. For $A=1, B=-1, p=1$ and $\alpha=\beta=0$, Theorem 1 agrees with Theorem 3 of Goel and Mehrok [3].

3. For $A=1$ and $B=-1$, Theorem 1 agrees with Theorem 2 of Aouf [2].

Theorem 2. For $f(z) \in C[A, B ; C, D, p, \beta, \alpha],|z| \leq r<1$,

$$
\begin{aligned}
& \left|\arg \frac{f^{\prime}(z)}{z^{p-1}}\right| \leq \\
& \left\{\begin{array}{l}
\left(\frac{C-D}{D}\right)(p-\alpha) \sin ^{-1}(D r)+\sin ^{-1} \frac{(A-B)(p-\beta) r}{p-[p B+(A-B)(p-\beta)] B r^{2}}, D \neq 0 \\
C(p-\alpha) r+\sin ^{-1} \frac{(A-B)(p-\beta) r}{p-[p B+(A-B)(p-\beta)] B r^{2}}, D=0 .
\end{array}\right.
\end{aligned}
$$

These inequalities are sharp.

Proof. From (2.1) and (1.2), we have

$$
\left|\arg \frac{f^{\prime}(z)}{z^{p-1}}\right| \leq\left|\arg \frac{f_{1}(z)}{z^{p}}\right|+|\arg p(z)|,
$$

$f_{1}(z) \in P^{*}[C, D, p, \alpha]$ and $p(z) \in P[A, B, p, \beta]$.

For $f_{1}(z) \in P^{*}[C, D, p, \alpha]$, we know $[1$, Theorem 2] that for $|z| \leq r<1$

$$
\left|\arg \frac{f_{1}(z)}{z^{p}}\right| \leq \begin{cases}\left(\frac{C-D}{D}\right)(p-\alpha) \sin ^{-1}(D r), & D \neq 0 \\ C(p-\alpha) r, & D=0 .\end{cases}
$$

Also for $p(z) \in P[A, B, p, \beta]$, we know $[1$, Theorem 4] that for $|z| \leq r<1$

$$
|\arg p(z)| \leq \sin ^{-1} \frac{(A-B)(p-\beta) r}{p-[p B+(A-B)(p-\beta)] B r^{2}} .
$$

Substituting (2.6) and (2.7) into (2.5) gives the result.

Equality is attained for $f(z) \in C[A, B ; C, D, p, \beta, \alpha]$ satisfying

$$
f^{\prime}(\dot{z})=\left\{\begin{array}{l}
p z^{p-1} \frac{1+\left[B+(A-B)\left(1-\frac{\beta}{p}\right)\right] \delta_{1} z}{1+B \delta_{1} z}\left(1+D \delta_{2} z\right) \\
p z^{p-1} \frac{1+\left[B+(A-B)\left(1-\frac{\beta}{p}\right)\right] \delta_{1} z}{1+B \delta_{1} z} e^{C(p-\alpha) \delta_{2} z}, D=0
\end{array}, D \neq 0,\right.
$$


where

$$
\begin{aligned}
\delta_{1}= & \frac{r}{z}\left\{\frac{-\left(\left[B+(A-B)\left(1-\frac{\beta}{p}\right)\right]+B\right) r}{1+\left[B+(A-B)\left(1-\frac{\beta}{p}\right)\right] B r^{2}}\right. \\
& \left.+i \frac{\sqrt{1-\left[B+(A-B)\left(1-\frac{\beta}{p}\right)\right]^{2} r^{2}} \sqrt{1-B^{2} r^{2}}}{1+\left[B+(A-B)\left(1-\frac{\beta}{p}\right)\right] B r^{2}}\right\}, r=|z|
\end{aligned}
$$

and

$$
\delta_{2}=\frac{r}{z} \cdot\left[-D r+i \sqrt{1-D^{2} r^{2}}\right]
$$

Remarks.

1. For $p=1$ and $\alpha=\beta=0$, Theorem 2 agrees with Theorem 2 of Silvia [19].

2. For $A=1, B=-1, p=1$ and $\alpha=\beta=0$, Theorem 2 agrees with Theorem 4 of Goel and Mehrok [4].

3. For $A=1$ and $B=-1$, Theorem 2 agrees with Theorem 3 of Aouf [2].

4. For $p=A=C=1$ and $B=D=-1$, Theorem 2, agrees with Theorem 4 of Silverman [18].

5. Choosing $p=A=C=1, B=D=-1$ and $\alpha=\beta=0$ in Theorem 2, we get the result due to Ogawa [14] and Krzyz [10].

\section{Hadamard product.}

The convolution or Hadamard product of two power series $f(z)=\sum_{n=0}^{\infty} a_{n} z^{n}$ and $g(z)=\sum_{n=0}^{\infty} b_{n} z^{n}$ is defined as the power series $(f * g)(z)=\sum_{n=0}^{\infty} a_{n} b_{n} z^{n}$. In order to obtain as subordination result linking $C[A, B ; C, D, p, \beta, \alpha]$ and $P[A, B, p, \beta]$ we need the following lemma:

Lemma 1 (Ruscheweyh and Sheil-Small, [17]). If $\psi(z)$ is regular in $U, \phi(z)$ and $h(z)$ are convex univalent in $U$ such that $\psi(z) \prec \phi(z)$, then $\psi(z) * h(z) \prec \phi(z) * h(z), z \in U$.

Theorem 3. If $f(z) \in C[A, B ; C, D, p, \beta, \alpha]$, then there exists $p(z) \in P[A, B, p, \beta]$ such that for all $s$ and $t$ with $|s| \leq 1,|t| \leq 1(s \neq t)$,

$$
\begin{gathered}
\frac{f^{\prime}(s z) p(t z) t^{p-1}}{f^{\prime}(t z) p(s z) s^{p-1}} \\
\prec \begin{cases}\left(\frac{1+D s z}{1+D t z}\right) & \left(\frac{C-D}{D}\right)(p-\alpha) \\
e^{C(p-\alpha)(s-t) z} & , D \neq 0,\end{cases}
\end{gathered}
$$


Proof. The proof is similar to the one given by Ruscheweyh [16], Goel and Mehrok [4] and Silvia [19].

We first consider the case when $D \neq 0$. We have

$$
\frac{z f^{\prime \prime}(z)}{f^{\prime}(z)}-\frac{z p^{\prime}(z)}{p(z)}-p+1=\left(1+\frac{z g^{\prime \prime}(z)}{g^{\prime}(z)}\right)-p
$$

$g(z) \in K[C, D, p, \alpha]$ and $p(z) \in P[A, B, p, \beta]$. Therefore,

$$
\frac{z f^{\prime \prime}(z)}{f^{\prime}(z)}-\frac{z p^{\prime}(z)}{p(z)}-p+1 \prec \frac{(C-D)(p-\alpha) z}{1+D z}
$$

where $\frac{(C-D)(p-\alpha) z}{1+D z}$ is convex, univalent in $U$. For $|s| \leq 1,|t| \leq 1,(s \neq t)$,

$$
h(z)=\int_{0}^{z}\left(\frac{s}{1-s u}-\frac{t}{1-t u}\right) d u
$$

is convex, univalent in $U$. (3.3) and (3.4) satisfy the conditions of Lemma 1, and therefore

$$
\left(\frac{z f^{\prime \prime}(z)}{f^{\prime}(z)}-\frac{z p^{\prime}(z)}{p(z)}-p+1\right) * h(z) \prec \frac{(C-D)(p-\alpha) z}{1+D z} * h(z) .
$$

Given any function $\ell(z)$ analytic in $U$ with $\ell(0)=0$, we have

$$
(\ell * h)(z)=\int_{t z}^{s z} \ell(u) \frac{d u}{u}, \quad z \in U
$$

By the application of (3.6) and (3.5) can be written as

$$
\int_{t z}^{s z}\left[\frac{u f^{\prime \prime}(u)}{f^{\prime}(u)}-\frac{u p^{\prime}(u)}{p(u)}-p+1\right] \frac{d u}{u} \prec(C-D)(p-\alpha) \int_{t z}^{s z} \frac{d u}{1+D u}
$$

from which (3.1) follows.

Similarly for $D=0$, we obtain (3.2).

Corollary 1 . If $f(z) \in C[A, B ; C, D, p, \beta, \alpha]$, then there exists a $p(z) \in P[A, B, p, \beta]$ and a Schwarz function $w(z) \in \Omega$ such that

$$
\frac{f^{\prime}(z)}{z^{p-1}}= \begin{cases}p(z)(1+D w(z))^{\left(\frac{C-D}{D}\right)(p-\alpha)}, & D \neq 0 \\ p(z) e^{C(p-\alpha) w(z)}, & D=0 .\end{cases}
$$

Proof. The result follows directly upon substituting $s=1$ and $t=0$ into Theorem 3 . 


\section{on SUBCLASSES OF P-VALENT Close-To-CONVEX FUnCtions}

Corollary 2. If $f(z)=z^{p}+\sum_{n=p+1}^{\infty} a_{n} z^{n} \in C[A, B ; C, D, p, \beta, \alpha]$, then

$$
\left|a_{p+1}\right| \leq \frac{p(C-D)(p-\alpha)+(A-B)(p-\beta)}{p+1}
$$

Proof. If $g \prec F$, then $\left|g^{\prime}(0)\right| \leq\left|F^{\prime}(0)\right|[12]$. From Corollary 1, we take $g(z)=$ $f^{\prime}(z) / z^{p-1} p(z)$ and

$$
F(z)= \begin{cases}(1+D z)^{\left(\frac{C-D}{D}\right)(p-\alpha)} & , D \neq 0 \\ e^{C(p-\alpha) z} & , D=0\end{cases}
$$

Then $g^{\prime}(0)=\frac{(p+1) a_{p+1}-c_{1}}{p}$ for $p(z)=p+\sum_{n=1}^{\infty} c_{n} z^{n}$ and $F^{\prime}(0)=(C-D)(p-\alpha)$. Therefore $\frac{(p+1)\left|a_{p+1}\right|-\left|c_{1}\right|}{p} \leq|(C-D)(p-\alpha)|$

and

$$
\begin{aligned}
\left|a_{p+1}\right| & \leq \frac{p|(C-D)(p-\alpha)|+\left|c_{1}\right|}{p+1} \\
& \leq \frac{p(C-D)(p-\alpha)+(A-B)(p-\beta)}{p+1} \quad \text { [1, Theorem 5] }
\end{aligned}
$$

as claimed.

\section{Coefficient Inequalities.}

We begin with coefficient inequalities for $K[C, D, p, \alpha]$.

Lemma 2. For $g(z)=z^{p}+\sum_{n=p+1}^{\infty} b_{n} z^{n} \in K[C, D, p, \alpha]$ and $\mu$ complex

$$
\left|b_{p+1}\right| \leq\left(\frac{p}{p+1}\right)(C-D)(p-\alpha)
$$

and

$$
\begin{aligned}
& \left|b_{p+2}-\mu b_{p+1}^{2}\right| \leq \frac{p}{2(p+2)}(C-D)(p-\alpha) \\
& \cdot \max \left\{1,\left|\frac{2 p(p+2)}{(p+1)^{2}} \mu(C-D)(p-\alpha)-[(C-D)(p-\alpha) p-D]\right|\right\}
\end{aligned}
$$

The result is sharp. 
Proof. For $g(z)=z^{p}+\sum_{n=p+1}^{\infty} b_{n} z^{n} \in K[C, D, p, \alpha]$, there exists a Schwarz function $w(z)=\sum_{n=1}^{\infty} \gamma_{n} z^{n} \in \Omega$ such that

$$
1+\frac{z g^{\prime \prime}(z)}{g^{\prime}(z)}=\frac{p+[p D+(C-D)(p-\alpha)] w(z)}{1+D w(z)}
$$

or

$$
1+\frac{z g^{\prime \prime}(z)}{g^{\prime}(z)}-p=\frac{(C-D)(p-\alpha) w(z)}{1+D w(z)} .
$$

Substituting of the series expansions and comparison of the coefficients leads to

$$
b_{p+1}=\left(\frac{p}{p+1}\right)(C-D)(p-\alpha) \gamma_{1}
$$

and

$$
b_{p+2}=\frac{p}{2(p+2)}(C-D)(p-\alpha)\left\{\gamma_{2}+[(C-D)(p-\alpha) p-D] \gamma_{1}^{2}\right\}
$$

Therefore,

$$
\left|b_{p+1}\right| \leq\left(\frac{p}{p+1}\right)(C-D)(p-\alpha)
$$

and

$$
\begin{aligned}
\delta_{p+2}-\mu b_{p+1}^{2}= & \frac{p}{2(p+2)}(C-D)(p-\alpha)\left\{\gamma_{2}+[(C-D)(p-\alpha) p-D)\right. \\
& \left.\left.-\frac{2 p(p+2)}{(p+1)^{2}} \mu(C-D)(p-\alpha)\right] \gamma_{1}^{2}\right\}
\end{aligned}
$$

We know [9] that for $w(z)=\sum_{n=1}^{\infty} \gamma_{n} z^{n} \in \Omega$, if $s$ is any complex number, then

$$
\left|\gamma_{2}-s \gamma_{1}^{2}\right| \leq \max \{1,|s|\}
$$

Equality is attained for $w(z)=z^{2}$ and $w(z)=z$.

Combining (4.4) and (4.5) yields the result, and since (4.5) is sharp, then (4.2) is also sharp.

Theorem 4. If $g(z)=z^{p}+\sum_{n=p+1}^{\infty} b_{n} z^{n} \in K[C, D, p, \alpha]$, then

$$
\left|b_{n}\right| \leq \frac{p}{n(n-p) !} \prod_{k=0}^{n-(p+1)}|(D-C)(p-\alpha)+D k|
$$

for $n \geq p+1$, and these bounds are sharp for all admissible $C, D$ and $\alpha$ and for each $n$. 
Proof. If $g(z)=z^{p}+\sum_{n=p+1}^{\infty} b_{n} z^{n} \in K[C, D, p, \alpha]$, then $\frac{z g^{\prime}(z)}{p}=z^{p}+\sum_{n=p+1}^{\infty} \frac{n}{p} b_{n} z^{n}$ is in $P^{*}[C, D, p, \alpha]$. But for $f_{1}(z)=z^{p}+\sum_{n=p+1}^{\infty} a_{n} z^{n} \in P^{*}[C, D, p, \alpha]$, we have $[1$, Theorem 3].

$$
\left|a_{n}\right| \leq \frac{1}{(n-p) !} \prod_{k=0}^{n-(p+1)}|(D-C)(p-\alpha)+D k|
$$

Then the result follows from (4.7) and replacing $a_{n}$ by $\frac{n}{p} b_{n}$.

For sharpness of (4.6) consider

$$
g^{\prime}(z)=p z^{p-1}(1-D \delta z)^{\left(\frac{C-D}{D}\right)(p-\alpha)},|\delta|=1, D \neq 0 .
$$

Theorem 5. For $f(z)=z^{p}+\sum_{n=p+1}^{\infty} a_{n} z^{n} \in C[A, B ; C, D, p, \beta, \alpha]$

$$
\left|a_{p+1}\right| \leq \frac{p(C-D)(p-\alpha)+(A-B)(p-\beta)}{p+1}
$$

and

$$
\begin{aligned}
& \left|a_{p+2}\right| \leq \\
& \left\{\begin{array}{l}
\frac{p}{2(p+2)}(C-D)(p-\alpha)+\frac{(A-B)(p-\beta)}{p+2}[(C-D)(p-\alpha)+1] \\
+\frac{p}{p+2}(C-D)^{2}(p-\alpha)^{2}+\frac{p}{p+2} B^{2},|(C-D)(p-\alpha) p-D| \leq 1 \\
\frac{p}{2(p+2)}(C-D)(p-\alpha)[(C-D)(p-\alpha) p-D] \\
+\frac{(A-B)(p-\beta)[(C-D)(p-\alpha)+1]}{p+2}+\frac{p}{p+2}(C-D)^{2}(p-\alpha)^{2} \\
+\frac{p}{p+2} B^{2},|(C-D)(p-\alpha) p-D|>1
\end{array}\right.
\end{aligned}
$$

Proof. There exists a $g(z)=z^{p}+\sum_{n=p+1}^{\infty} b_{n} z^{n} \in K[C, D, p, \alpha]$ and a Schwarz function $w(z)=\sum_{n=1}^{\infty} \gamma_{n} z^{n} \in \Omega$ such that

$$
\frac{p f^{\prime}(z)}{g^{\prime}(z)}=\frac{p+[p B+(A-B)(p-\beta)] w(z)}{1+B w(z)}, z \in U
$$

Comparing series expansions, we see

$$
a_{p+1}=b_{p+1}+\frac{(A-B)(p-\beta)}{p+1} \gamma_{1}
$$


and

$$
\begin{aligned}
a_{p+2}= & b_{p+2}+\frac{p+1}{p(p+2)}(A-B)(p-\beta) b_{p+1} \gamma_{1} \\
& +\frac{(A-B)(p-\beta)}{p+2}\left\{\gamma_{2}-B \gamma_{1}^{2}\right\}+\frac{(p+1)^{2}}{p(p+2)} b_{p+1}^{2}-\frac{p}{p+2} B^{2} \gamma_{1}^{2} .
\end{aligned}
$$

The bound for $\left|a_{p+1}\right|$ follows from Lemma 2. Applying (4.5) and Lemma $2(\mu=0)$ to (4.12), we have

$$
\begin{aligned}
\left|a_{p+2}\right| \leq & \frac{p}{2(p+2)}(C-D)(p-\alpha) \max \{1,|(C-D)(p-\alpha) p-D|\} \\
& +\frac{(A-B)(p-\beta)(C-D)(p-\alpha)}{p+2}+\frac{(A-B)(p-\beta)}{p+2} \max \{1,|B|\} \\
& +\frac{p}{p+2}(C-D)^{2}(p-\alpha)^{2}+\frac{p}{p+2} B^{2} \\
= & \frac{p}{2(p+2)}(C-D)(p-\alpha) \max \{1,|(C-D)(p-\alpha) p-D|\} \\
& +\frac{(A-B)(p-\beta)}{p+2}[(C-D)(p-\alpha)+1]+\frac{p}{p+2}(C-D)^{2}(p-\alpha)^{2} \\
& +\frac{p}{p+2} B^{2} .
\end{aligned}
$$

\section{References}

[1] M.K. Aouf, "On a class of $p$-valent starlike functions of order $\alpha$," International J. Math. Sci. 10(1987), no. 4, 733-744.

[2] M.K. Aouf, "On a class of $p$-valent close-to-convex functions of order $\beta$ and type $\alpha$," International J. Math. Math. Sci. 11(1988), no. 2, 259-266.

[3] R.M. Goel and B.S. Mehrok, "On the coefficients of a subclass of starlike functions," Indian J. Pure Appl. Math., 12(1981), 634-637.

[4] R.M. Goel and B.S. Mehrok, "On a class of close-to-convex functions", Indian J. Pure Appl. Math., 12(1981), 648-658.

[5] R.M. Goel and B.S. Mehrok, "Some invariance properties of a subclass of close-to-convex functions," Indian J. Pure Appl. Math., 12(1981), 1240-1249.

[6] E.G. Goluzian, "On the coefficients of a class of functions regular in a disk and having an integral representation in it", J. of Soviet Math. (2) 6, (1974), 606-617.

[7] W. Janowski, "Some extremal problems for certain families of analytic functions", Ann. Polon. Math., 28(1973), 297-326.

[8] W. Kaplan, "Close-to-convex schlicht functions," Mich. Math. J., 1(1952), 169-185.

[9] F.R. Keogh and E.P. Merkes, "A coefficient inequality for certain classes of analytic functions," Proc. Amer. Math. Sco., 20(1969), 8-12.

[10] J. Krzyz, "On the derivative of close-to-convex functions," Coll. Math., 10(1963), 139-142.

[11] R.J. Libera, "Some radius of convexity problems," Duke Math. J. 31(1964), 143-158.

[12] Z. Nehari, "Conformal Mapping", McGraw-Hill Book Co., Inc., New York (1952).

[13] R. Mazur, "On a subclass of convex functions," Zeszyty Nauk. Politech. todz. Mat. 13(1981), 15-20.

[14] S. Ogawa, "A note on close-to-convex functions," J. Nara Gakugei Univ., 8(1959), 9-10. 
[15] M.S. Robertson, "On the theory of univalent functions," Ann. of Math., 37 (1936), 169-185.

[16] St. Ruscheweyh, A. Subordination theorem for $\phi$-like functions," J. London Math. Soc., 13(1976), 275-280.

[17] St. Ruscheweyh and T. Shell-Small, "Hadamard product of schilcht functions and the Polyar Schoenberg conjecture," Comment. Math. Helv., 48(1973), 119-135.

[18] H. Silverman, "Convexity theorems for subclasses of univalent functions," Pacific J. of Maths., vol. 64, No. 1(1976), 253-263.

[19] E.M. Silvia, "Subclasses of close-to-convex functions," Internat. J. Math. Math. Sci. Vol. 6, No. 3 (1983), 449-458.

Department of Mathematics, Faculty of Science, University of Mansoura, Mansoura, Egypt. 